\title{
Virtual Engineering Approach To Developing Selective Harvest Technologies
}

\section{ASAE Annual International Meeting}

Kevin L. Kenney

Christopher T. Wright

Kenneth M. Bryden, Ph.D.

\author{
July 2005
}

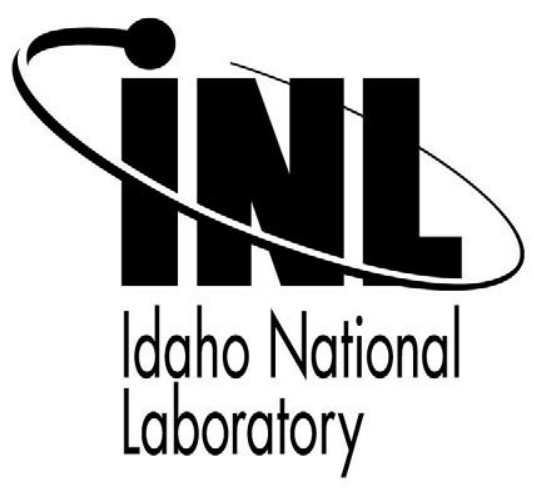

This is a preprint of a paper intended for publication in a journal or proceedings. Since changes may not be made before publication, this preprint should not be cited or reproduced without permission of the author. This document was prepared as an account of work sponsored by an agency of the United States Government. Neither the United States Government nor any agency thereof, or any of their employees, makes any warranty, expressed or implied, or assumes any legal liability or responsibility for any third party's use, or the results of such use, of any information, apparatus, product or process disclosed in this report, or represents that its use by such third party would not infringe privately owned rights. The views expressed in this paper are not necessarily those of the United States Government or the sponsoring agency. 


\title{
Virtual Engineering Approach to Developing Selective Harvest Technologies
}

\section{Kevin L. Kenney}

Idaho National Laboratory, P.O. Box 1625, Idaho Falls, ID 83415-2210, kevin.kenney@inl.gov

\section{Christopher T. Wright}

Idaho National Laboratory, P.O. Box 1625, Idaho Falls, ID 83415-2210, christopher.wright@inl.gov

\section{Kenneth M. Bryden, Ph.D.}

lowa State University, 3030 H. M. Black Engineering Bldg. Ames, IA 50011-2160, kmbryden@iastate.edu

\author{
Written for presentation at the \\ 2005 ASAE Annual International Meeting \\ Sponsored by ASAE \\ Tampa Convention Center \\ Tampa, Florida \\ 17 - 20 July 2005
}

\begin{abstract}
Agricultural crop residues (e.g., straw and stover) are a current focus for bioenergy feedstocks, with new technologies being developed to improve the economics of bioenergy production. Among the emerging technologies focused on feedstock engineering is the selective harvest concept. Due to the complexity of the biomass separations required for addressing the challenges and requirements of selective harvest, high fidelity models and advanced experimental methods that allow observation and measurement of the physical system are needed. These models and methods were developed and include computational fluid dynamics (CFD) modeling to simulate the cleaning shoe of a grain combine and a particle image velocimetry (PIV) technique to quantitatively and qualitatively characterize the cleaning shoe performance. While these techniques alone can be sufficient engineering and analysis tools for developing selective harvest technologies, this paper presents a new methodology, Virtual Engineering (VE), that integrates the CFD and PIV data into a virtual environment, where the data is coupled with the geometric model of a grain combine to provide a virtual representation of the cleaning shoe performance. Using VE visualization capabilities, the CFD and PIV data can be viewed in the context of the physical system for an interactive evaluation of characteristics and performance. This paper also discusses the concepts of additional VE tools that are being developed to provide necessary visualization, simulation and integration functionality.
\end{abstract}


Keywords. selective harvest, feedstock engineering, virtual engineering, virtual reality, computational engineering, computational fluid dynamics, CFD, particle image velocimetry, PIV. 


\section{Introduction}

The sustainability and economics of bioenergy production are significantly dependent on an integrated feedstock assembly system that is capable of providing the necessary large supply of low-cost, high-quality lignocellulosic biomass to biorefineries that produce fuels, combined heat and power, chemicals and other materials. The feedstock assembly system is taking shape with the development of advanced technologies and methods for harvesting and collection, preprocessing, bulk handling and storage, which reduce feedstock costs and improve feedstock quality.

Although the future of bioenergy will require a wide variety of feedstock streams as inputs to biorefineries and power plants (Biomass Technical Advisory Committee, 2002), this research is currently focused on the most available, sustainable, and cost effective feedstocks - agricultural crop residues - to meet the near-term feedstock goals (DOE, 2002). Of the agricultural residues, corn stover and wheat straw have been identified as the most likely high-volume lignocellulosic feedstocks available (DOE, 2002). Agricultural residues, however, present some unique feedstock assembly challenges requiring the development of new technologies tailored specifically to these feedstocks.

A technology that has significant potential for reducing harvesting and collecting costs of agresidue feedstocks and improving the feedstock quality is selective harvest. The objective of the selective harvest strategy is to simultaneously separate and collect multiple components of the crop for efficient harvest of diversified agriculture products that are utilized for their highest value end use. The advantages of this approach are realized in 1) increasing harvest efficiency by eliminating additional collection and separation processes, 2) maximizing the agricultural products from a single crop, 3) utilizing each component according to its highest value end-use (this includes leaving biomass in the field for soil sustainability), 4) minimizing material handling, 5 ) minimizing residue loss associated with depositing it on the ground followed by subsequent collection and 6) eliminating bioenergy feedstock contamination due to pick-up of dirt and debris while collecting it from the ground. The challenges associated with this are 1) implementing additional separations without affecting the efficient harvest and collection of grain (this includes both the efficient cleaning and maintaining the throughput of the harvester), 2) developing high fidelity separations of particles that do not have physically distinct properties, 3 ) implementing the additional separations in the confines of a combine and 4) collecting the additional product streams.

Designing selective harvest technologies to meet these challenges requires the use of advanced engineering techniques, which are typically not employed for engineering biomass separations in current combine designs. Low fidelity engineering models, experience and trialand-error techniques have generally been adequate for biomass separations in modern combines. Due to the significant disparity of the physical properties of grain and material-otherthan-grain (MOG), or residues, high fidelity models (e.g., computational fluid dynamics (CFD)) and sophisticated experimental measurement and observation techniques have not been necessary. However, selective harvest technologies capable of separating components of the MOG without affecting grain harvest are more difficult due to the similarity of the components' physical properties. For these systems high fidelity models and advanced experimental methods that can be used to determine apriori the important aspects of the flow for guiding, refining and verifying the computational models are essential design tools.

This paper describes a Virtual Engineering (VE) approach to developing biomass separation methods for accomplishing selective harvest objectives. Virtual engineering is an emerging technology that is not currently capable of solving this problem. However, the approach 
discussed in this paper illustrates the integration of key components of a virtual engineering model, and discusses some of the virtual engineering tools that are being developed to build the VE capabilities required to solve this problem.

\section{Background}

The illustrations in Figures 1-3 are intended to help understand and put into context the equipment engineering focus of selective harvest. Figure 1 is an illustration of a rotary combine that is used as the base harvest system for integration of selective harvest technologies. The cutaway view shows the separation and cleaning chamber where the grain is separated (threshed) from the plant and then cleaned to remove the grain from the crop residues. A detailed illustration identifying the various components of the separation and cleaning systems is shown in Figure 2, and the components are identified as follows: A-Feeder, B-Twin Rotors, CConcaves, D-Grain Pan, E-Upper Sieve, F-Lower Sieve, G-Cleaning Fan, H-Discharge Beater and I-Separation Grate. The cleaning shoe of a grain combine, which encompasses the grain pan, upper sieve, lower sieve and cleaning fan, separates the grain from the crop residues, and is illustrated in Figure 3. The cleaning shoe incorporates a combination of mechanical shaking to disperse the biomass, and forced air to fluidize the less dense MOG while allowing the more dense grain to fall through the sieves (Deere and Company, 1967, 1987). Incorporating more sophisticated biomass separations for selective harvest will require detailed understanding of cleaning shoe performance to insure that the selective harvest methods do not adversely affect cleaning shoe performance and take advantage of cleaning shoe performance for accomplishing residue separations.

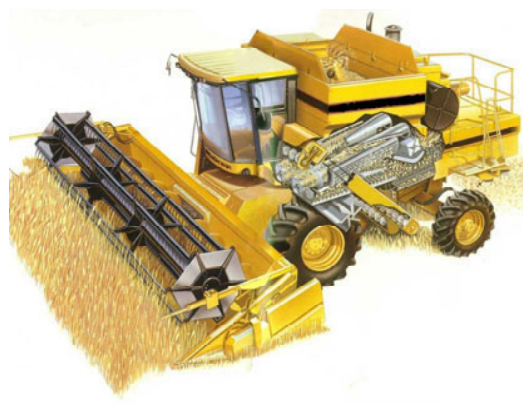

Figure 1: A modern grain combine represents the stateof-the-art in biomass separations.

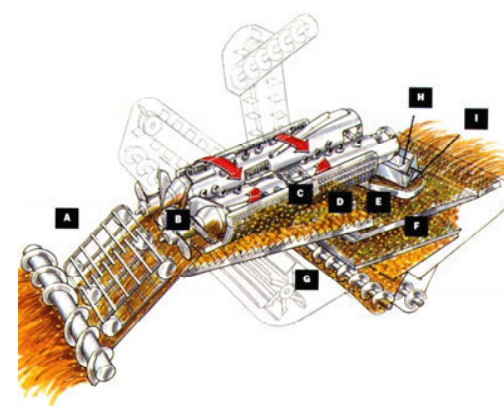

Figure 2: Combine separation, cleaning and grain handling systems.

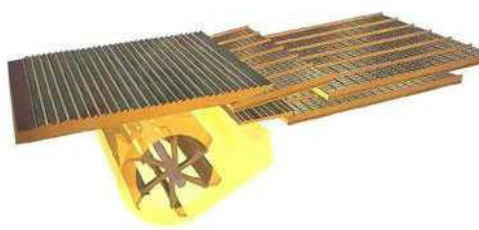

Figure 3: Combine cleaning shoe that is the focus of experimental and numerical characterization.

From a fluid dynamics viewpoint, the cleaning shoe biomass separation process can be characterized as gas/solid multi-phase flow with impacting moving boundaries. The physics of these types of flows is very complicated and presents unique challenges for developing methods to characterize these flows (Chao et al., 1998). High fidelity models and sophisticated experimental techniques offer the best opportunity for understanding these flows in sufficient detail to engineer advanced separations of selective harvest.

\section{Numerical Modeling}

CFD modeling is a well-proven technique for analyzing and visualizing flow fields. However, simulating the biomass separation processes with CFD models requires incorporating all of the 
complex geometric and flow field parameters inherent in the combine separation process. Building a model that adequately incorporates these parameters is, therefore, very complicated, and at the forefront of current CFD capabilities. Nevertheless, advanced CFD models have been developed, which incorporate cutting edge methodologies to adequately model the effects of air flow and mechanical agitation on the performance of the combine cleaning shoe.

Two separate CFD software packages, ANSYS CFX-5 (ANSYS, 2004) and Fluent 6 (Fluent, 2004), were used to develop the cleaning shoe models. Since these two packages are fundamentally different in the way they solve the fluid flow equations and apply the moving boundary conditions, they provide additional independent verification of the flow field. Figures 4 and 5 show images of the single-phase velocity contours resulting from both software packages. These results compare well to each other, showing similar velocity magnitudes and flow patterns. The behavior of the flow in the vicinity of the sieves is of primary interest in the separation model. In fact, this region of the flow will be a critical part to the development of twophase models as it affects the actual separation of the MOG components in the air column.

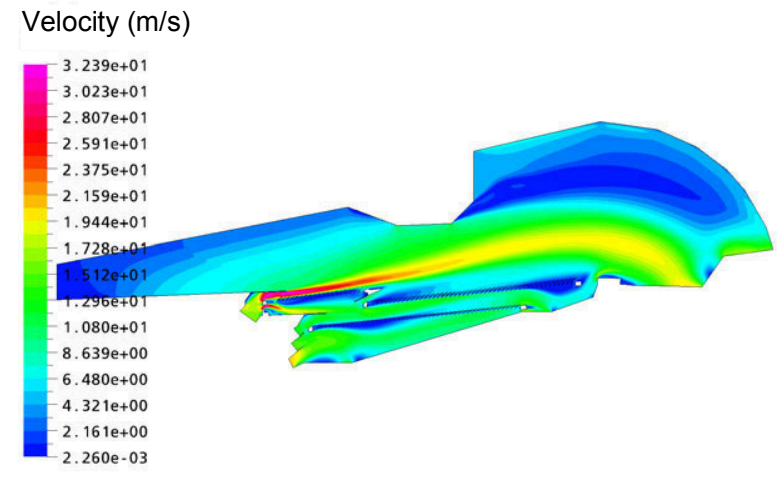

Figure 4: CFD model results from CFX 5.

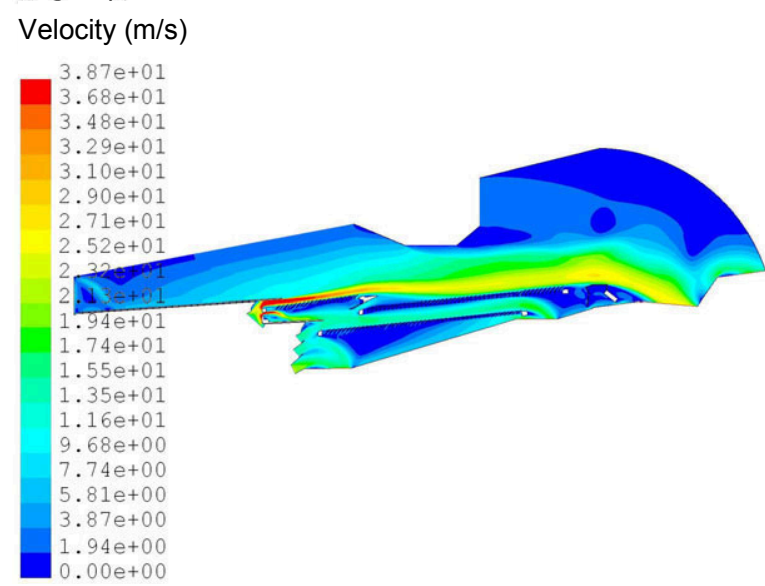

Figure 5: CFD model results from Fluent 6.

These results represent a fundamental look at the single-phase flow of air through the cleaning system. These models provide a more complete understanding of the cleaning shoe performance in terms of the air stream flow patterns and characteristics, the separation mechanisms of grain and MOG and the potential effects the air column might have on the fractionation of the residue in the air stream above the cleaning shoe. This understanding, though limited to a single-phase view of the flow, is useful in identifying opportunities and developing techniques for separating the residues in the air column, insuring selective harvest modifications do not adversely affect cleaning shoe performance, and identifying possible ways of improving the cleaning process for the collection of grain.

The primary purpose of the CFD models is to provide a fundamental look at the complex and non-intuitive flow of air through a combine cleaning system. This a primary input to the Virtual Engineering model (described below) as it is both descriptive, for evaluating current cleaning shoe performance, and predictive, for evaluating the effects of selective harvest designs on cleaning shoe performance. 


\section{Experimental Measurements}

Experimental measurements of the cleaning shoe air stream behavior were performed using a Particle Image Velocimetry (PIV) technique. This technique is used to measure the in-plane two-component velocity field of a fluid flow (Bachalo et al., 1996; Grant, 1997; Hasaan, 1998; Westerweel, 1997). The configuration of a typical PIV system is illustrated in Figure 6. A laser light sheet illuminates a plane in the flow field and a camera, which is oriented perpendicular to the light sheet plane, records the positions of particles entrained in the flow at each instant the light sheet is pulsed. A pair of single exposure images is required for PIV data processing, which consists of determining the average displacement of the exposed particles between subsequent pulses of the light sheet. Knowledge of the time interval between light sheet pulses then permits computation of the flow velocity. Dividing the images into many interrogation regions produces a 2-D velocity map where the velocity within an interrogation region is accurately described by a single velocity vector. Flow properties such as vorticity, strain rates, turbulence and other higher order flow statistics can also be obtained.

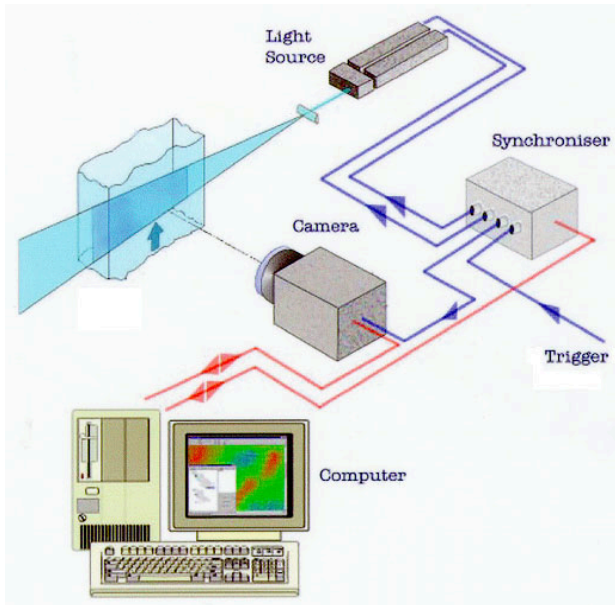

Figure 6: Schematic of a typical PIV system.

In order to visualize the air stream flow field, tracer particles were injected into the flow. The process of injecting tracer particles into the flow field analyzed by PIV is referred to as artificial seeding, and is a common technique for imaging single-phase flows. Artificial seeding is achieved by introducing small neutrally buoyant particles upstream of the interested region. The particles are then entrained into the flow field and conform to the actual flow patters in the fluid. For the best quality PIV measurement, the seed particles must be injected into the flow shortly before the air stream enters the test section. Furthermore, the injection has to be done without significantly disturbing the flow and in a way and location that ensures homogeneous distribution of the tracers in the flow. The best results were obtained by injecting the seed particles directly into the cleaning fan.

The objective of the PIV testing was to provide both a quantitative and qualitative analysis of the single-phase airflows in the separation chamber of a grain combine for CFD model verification. To be consistent with the 2-D CFD models, which were located along the centerline of the combine separation chamber, a test matrix was constructed to map the airflow along the centerline as well. Since each individual image covered an $8 \times 8$-inch area, many tests were run to construct the entire PIV map. This PIV map is shown in Figure 7 where the velocity vectors represent the direction of the airflow above the cleaning shoe. As demonstrated in this figure, PIV allows both quantitative verification through analysis of the vectors and qualitative verification through visualization of the flow field structures.

\section{Virtual Engineering Approach}

Virtual engineering (VE) is the concurrent application of an integrated set of decision-making tools for addressing a wide range of engineering issues including design, cost, analysis, safety, maintenance, operation and development of a product or system (Xiao, Bryden, Englebrecht, et al., 2004; Xiao, Bryden, 2004). A virtual engineering model typically consists of a geometric model, related engineering models and experimental data. Three categories of engineering 


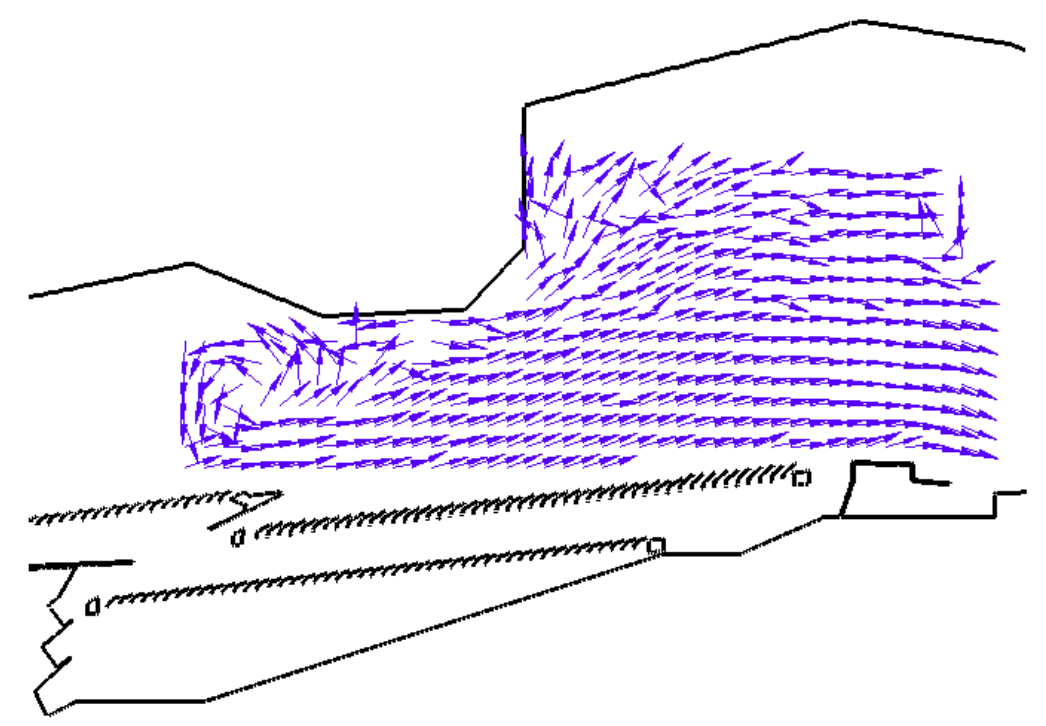

Figure 7: PIV map of single-phase air flow in a grain combine separation chamber

models exist: a) typical analysis and numerical simulation models, e.g., finite element analysis (FEA), CFD, etc.; b) mathematical formulations of product characteristics or properties, e.g., spreadsheet models of cost, safety, etc.; and c) empirical formulations obtained from experimental techniques. The goal of VE is to provide a user-centric, first person perspective of a particular problem, where the user can interact with the VE model in a natural way using a wide range of tools that aid in making engineering decisions.

Virtual engineering is a developing technology that will ultimately encompass a broad range of tools for integrated decision making. This particular project is interested in a few specific tools necessary for engineering selective harvest technologies. These include visualization tools for effectively utilizing CFD and experimental data, simulation tools that allow running design scenarios in the virtual environment and integration tools for coupling VE with a CFD solver.

\section{Visualization Tools}

Visualization tools are particularly useful for coupling computational or experimental data with a geometric model to provide a high-fidelity representation of the overall system. The virtual representation allows users to "walk through" the system and observe how it works, providing an experience similar to that of actually inspecting the physical system or device. Additional visualization tools allow the user to add and overlay layers of information (e.g., CAD drawings, CFD results, FEA results, or data from physical measurements) for an integrated evaluation of the system, or to trace flows to reveal complex or intricate flow patterns. This visualization capability is a powerful and useful engineering tool. CFD data (usually velocity, pressure, and temperature) fit well in this coupling process since they are readily transformable into a threedimensional workspace and thus can be easily visualized in a three-dimensional world. Figures 8-10 illustrate the visualization of the CFD model discussed above, in the VE environment.

The visualization capabilities of VE also provide the coupling of computational and experimental data in a common platform to enable validation of either the computational model or the experimental technique. Traditionally, validation using discrete data sets is accomplished by manually comparing the different data sets. However, VE offers a unique interaction with the models that provide opportunities for a much broader and more useful coupling of multiple data 


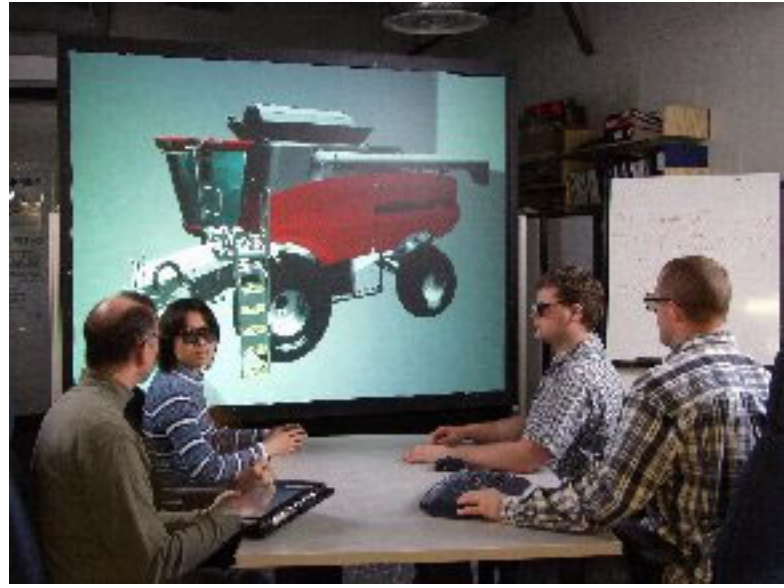

Figure 8: VE model of grain combine

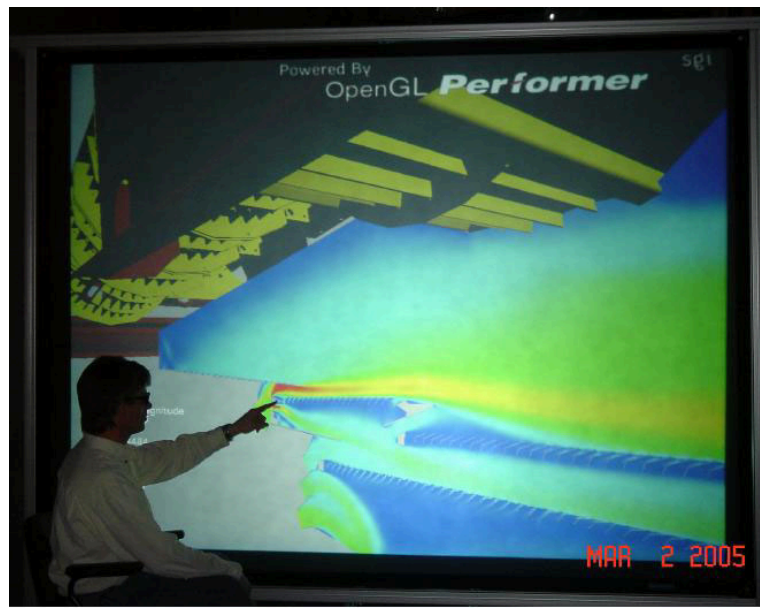

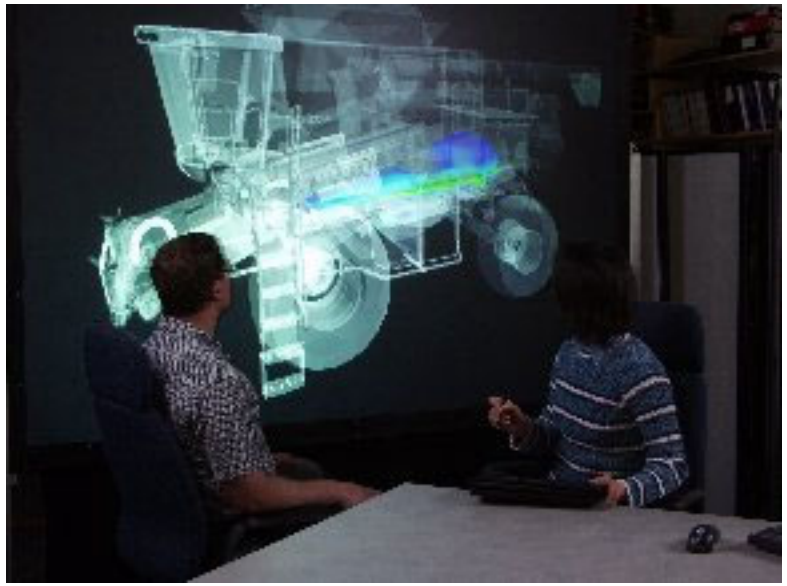

Figure 9: VE model of combine showing the CFD data set in the separation chamber

Figure 10: CFD data set in VE model

sets. This interaction includes tools for model validation as well as tools to interrogate the experimental data set in order to refine the boundary conditions of the CFD model. This capability is unique since, traditionally, boundary conditions are established using point source measurements, or in cases where these measurements could not or were not obtained, the boundary conditions are assumed. Thus, using VE interrogation tools, CFD boundary conditions can be retrieved from experimental data sets, automatically inserted into the CFD model, and used to recompute new CFD results all within the VE environment.

\section{Simulation Tools}

The development of virtual engineering tools is not limited to an environment where only data interrogation and investigation occur but also includes an environment where high-level engineering decision-making occurs. This capability requires interactive simulation tools that allow running design scenarios in the virtual environment. Coupling CAD and computational modeling functionality is key to these interactive simulation tools in that they allow an engineer 
to modify the system geometry and evaluate the system response all within the virtual environment.

\section{Integration Tools}

A primary component to engineering decision making using VE simulation is the integration of $\mathrm{CAD}$ and computational models in the virtual environment - not from a visualization and investigation perspective, but from an execution perspective. Integration tools for coupling VE with a CFD solver allow the CFD solver to be executed via the virtual environment by a nonCFD user. This includes much more than an executable link between the VE environment and CFD software, but allows the user full control over CFD parameters such as domain boundaries, boundary conditions, transient events, or output formats, just to name a few. This integration capability is critical if VE is to truly allow a user to have full decision making control. For example, the functionality of current high fidelity modeling, which is computationally intensive, is generally limited to a specific design case and predetermined outputs rather than allowing a user to design and optimize on the fly. In many cases, this limitation can make it much more efficient to take an experimental or trial-and-error approach rather than to wait for CFD results, especially when CFD is used for optimization and an iterative process is required. However, without the high-fidelity model, an optimum design may not be achieved. Therefore, techniques for reducing CFD run-time are required to transform the models into useful design and optimization tools. Various methodologies have been attempted for this purpose (Fan, 1998; Foster et al., 1997; McCorkle et al., 2003; Poloni et al., 2000; Zha et al., 1997), and the tools that will be developed to accomplish this in VE will integrate one or more of these techniques.

The software foundation encompassing the virtual engineering and decision-making process is in a package called VE-Suite (Bryden, 2005). VE-Suite is structured in such a manner that separate modules can be created that will allow data from various sources, as described above, to be integrated into a single environment (i.e. virtual conference room, multi-wall immersive environment, desktop PC) to be used in the virtual engineering decision making process.

\section{Conclusion}

Due to the complex nature of biomass separations, high fidelity models and advanced experimental techniques have been developed to address the challenges and requirements of the selective harvest concept. These models and techniques include CFD models and PIV techniques that quantitatively and qualitatively characterize the cleaning shoe performance. Alone, CFD and PIV are capable engineering and analysis tools for developing many of the selective harvest technologies. However, a new methodology, Virtual Engineering, is being developed that integrates the CFD and PIV data into a 3-D virtual environment, where the data are coupled with the combine geometry to provide a virtual representation of the cleaning shoe performance. The VE visualization capability allows the CFD and PIV data to be viewed in context of the physical system for an interactive evaluation of its characteristics and performance. In addition, advanced visualization, simulation and integration tools are being developed to actively couple the engineering and analysis tools in the virtual environment. While the development of these tools will improve VE capabilities to facilitate engineering decision making for the selective harvest application, they also advance the state of VE development for engineering of complex fluid systems in general.

VE uses the same engineering and analysis tools as traditional engineering approaches, but by actively coupling these tools in a common environment, VE takes integrated decision making to a whole new level. Traditional engineering approaches involve the interaction of subject matter experts who impose their requirements on the design (function, aesthetics, cost, manufacturing, 
etc.). This approach requires that the engineering and design processes become iterative and involve every subject matter expert in order for the design to converge and meet all the requirements. By integrating all of the decision making tools (geometric models, CFD models, structural models, cost models, etc.) into the VE model, and presenting the data in a format most pertinent to the decision being made, all relevant design issues can be evaluated simultaneously. Furthermore, the ability to evaluate and compare different design scenarios through a 3-D virtual representation of the product/system, which includes not only graphics but experimental and numerical data as well, is a capability unmatched by traditional approaches. This integrated approach greatly simplifies the engineering design process, thus, reducing time to market and development costs.

\section{Acknowledgements}

The authors thank Steve Kirstukas, Doug McCorkle and Sunil Suram of the lowa State University Virtual Reality Application Center (VRAC) for their ongoing contributions to this work including the development of VE-Suite and many of the VE tools discussed in this paper.

This work is supported by the US Department of Energy, Assistant Secretary for Energy Efficiency and Renewable Energy (EERE) under DOE Idaho Operations Office Contract DEAC07-05ID14517.

\section{References}

ANSYS. 2004. CFX. Ver. 5.7. Canonsburg, PA: ANSYS, Inc.

Bachalo, W., N. Chigier, W. J. Brown, and R. Reitz. 1996. Spray Technology Short Course. Pittsburgh, PA.: Norman Chigier, Mechanical Engineering Dept., Carnegie Mellon University.

Biomass Technical Advisory Committee. 2002. Vision for Bioenergy \& Biobased Products in the United States. Washington, D.C.: U.S. Department of Energy. Available at http://www.bioproducts-bioenergy.gov/pdfs/BioVision_03_Web.pdf. Accessed 06 May 2005.

Bryden K. M. 2005. VE-Suite. Available at http://www.vrac.iastate.edu/ kmbryden/VE-Suite.htm Accessed 09 May 2005.

Chao, Z., and L. S. Fan. 1998. Multiphase Flow: Gas/Solid. In The Handbook of Fluid Dynamics. Chapter 18. R.W. Johnson and C. Zhu, eds. Boca Raton, FL, CRC Press.

Deere and Company. 1976, 1987. Combine Harvesting. In Fundamentals of Machine Operation (FMO). Moline, IL.

DOE. 2003. Roadmap for Agriculture Biomass Feedstock Supply in the United States. DOE/NEID-11129. Washington, D.C.: U.S. Department of Energy.

Fan, H. Y. 1998. Inverse design method of diffuser blades by genetic algorithms. In Proc. Inst. Mech. Engrg. A(212): 261-268.

Fluent . 2004. Fluent, Ver. 6.2.16 Lebanon, NH: Fluent, Inc.

Foster, G. F., and G. S. Dulikravich. 1997. Three-dimensional aerodynamic shape optimization and gradient search algorithms. In J. Spacecraft Rockets. 34:36-42.

Grant, I. 1997. Particle image velocimetry: a review. Proc. Institution of Mechanical Engineers, 211(c): 55-76.

Hasaan, Y. A. 1998. Multiphase Flow Measurements Using Particle Image Velocimetry. In The Handbook of Fluid Dynamics, 36-1 to 36-15. R.W. Johnson and C. Zhu, eds. Boca Raton, FL, CRC Press. 
McCorkle, D. S., K. M. Bryden, and C. G. Carmichael. 2003. A new methodology for evolutionary optimization of energy systems. In Comput. Methods Appl. Mech. Engrg. 192(44-46):5021-5036.

Poloni, C., A. Giurgevich, L. Onesti, and V. Pediroda. 2000. Hybridization of a multi-objective genetic algorithm, a neural network and a classical optimizer for a complex design problem in fluid dynamics. In Comput. Methods Appl. Mech. Engrg. 186:403-420.

Westerweel, J. 1997. Fundamentals of digital particle image velocimetry. MeasurementScience-\&-Technology, 8(12): 1379-1392.

Xiao, A. and K. Bryden. 2004. Virtual Engineering: A Vision of Next-Generation Product Realization Using Virtual Reality Technologies. DETC2004/CIE-57698. In Proc. 2004 ASME Design Engrg. Technical Conf., Comput. in Engrg. Conf. New York, NY: ASME.

Xiao, A., K. Bryden, J. Englebrecht, and D. McCorkle. 2004. Acceleration Methods in the Interactive Design of a Hydraulic Mixing Nozzle Using Virtual Engineering Tools. IMECE2004-61821. In Proc. 2004 ASME International Mech. Engrg. Congress. New York, NY: ASME.

Zha, G., D. Smith, M. Schwabacher, K. Rasheed, A. Gelsey, D. Knight, and M. Haas. 1997. High performance supersonic missile inlet design using automated optimization. In J. Aircraft. 34: 697-705. 